\title{
Diagnostic optique en mécanique des fluides : sophistication et diversification au menu 2011
}

En juillet 2008, nous écrivions dans Photoniques n 36 : «La caractérisation des fluides par moyens optiques se porte bien malgré son âge». Nous évoquions également la percée significative des pays asiatiques dans ce domaine d'activité. Qu'en est-il trois ans plus tard? Pas de rupture scientifique ou technologique, mais comme le bon vin, la discipline s'est encore bonifiée avec le temps. Quant à l'Asie, comme dans bien d'autres domaines, elle confirme sa place de leader.

$>$ Jean-Pierre PRENEL

Université de Franche-Comté / Femto-ST

Président d'honneur Club SFO «Fluvisu»

\section{Les moyens optiques \\ toujours en vedette}

\section{Chronique d'une mort annoncée, fin de l'épisode}

La cause est entendue, la prévision «à la Nostradamus » d'une disparition programmée pour le début du xxI ${ }^{\mathrm{e}}$ siècle de l'expérimentation en mécanique des fluides au profit des seuls modèles s'est révélée fausse. Bien sûr, les modèles ont fait des progrès considérables, mais rares sont les scientifiques radicaux qui nient encore la nécessité d'un calage et d'une validation par des moyens expérimentaux. D'ailleurs, les modèles purement prédictifs, en science comme en économie ou en finance, ont montré, après une période d'euphorie, la nécessité d'une approche plus modeste et plus rationnelle. La science implique toujours le doute...

L'expérimentation, loin de disparaître, a donc encore élargi sa place au soleil depuis notre dernier bilan. Les mêmes progrès techniques qui ont fait le bonheur des modélisateurs ont permis également la sophistication des moyens de mesure, plus précis, plus rapides mais aussi de plus en plus fiables.

Indépendamment de la polémique très médiatique et politique qui accompagne la chasse au $\mathrm{CO}_{2}$, la prise de conscience, lente mais réelle, de la nécessité d'économiser l'énergie s'est révélée être l'une des puissantes motivations des chercheurs et desingénieurs utilisant la mécanique des fluides, puisque cette discipline constitue l'une des bases de l'énergétique. Même si des progrès restent à réaliser, il suffit de comparer à prestations égales la consommation des véhicules thermiques des années 1980 et celle de la génération actuelle pour constater que le progrès fait son œuvre. Dans ce domaine, l'association étroite entre expérimentation et modélisation constitue une réussite évidente.

\section{Pourquoi toujours plus d'optique dans cette évolution?}

La mécanique des fluides a longtemps été le domaine de prédilection des capteurs localisés, utilisés individuellement ou à la rigueur en réseaux, linéaires ou plus rarement matriciels. Purement mécaniques, thermiques ou thermomécaniques, ils restent d'actualité dans de nombreux domaines industriels. Les sondes de Pitot, révélées malheureusement au grand public à travers un événement rare mais particulièrement tragique, rendent de grands services depuis leur introduction en aéronautique, après avoir été proposées, dès 1732, par leur inventeur (français) pour mesurer la vitesse des liquides.

Les avantages de ces capteurs sont bien identifiés: le plus souvent simples, robustes et peu coûteux, ils sont aisément couplés aux moyens électroniques et infor- matiques modernes de la métrologie. Ce mariage leur a donné une seconde jeunesse. Leurs inconvénients ont ouvert la porte à l'optique très tôt dans I'histoire de la mécanique des fluides:

- par nature, ils sont perturbants, générant tourbillons et sillages; la première réponse fut la miniaturisation, très développée dès les années 1980, mais vite compensée par la réduction d'échelle de certains écoulements : microfluidique, et aujourd'hui tendance vers la nanofluidique ;

- par leur principe même, ils donnent des informations locales, limitation difficilement combattue par leur organisation en réseaux, le balayage mécanique dans l'espace n'apportant qu'une réponse partielle, limitée à l'étude des phénomènes stationnaires ;

- par leur conception, ils résistent avec peine à des écoulements rapides : vibrations, voire rupture.

La réponse passant par une amélioration du comportement mécanique se traduit le plus souvent par une augmentation de volume et donc une aggravation du caractère perturbant. Ils restent toutefois d'actualité pour les écoulements à moyenne ou grande échelle. Les sondes de Pitot, déjà citées, restent ainsi présentes sur les aéronefs, mais aussi sur les monoplaces de Formule 1.

C'est pourquoi les pionniers de la mécanique des fluides ont cherché à remplacer les capteurs par des faisceaux de lumière, pratiquement sans interaction avec le fluide et permettant I'observation simultanée d'un plein champ. $\gg$ 
Reprenant les travaux de Foucault, destinés à mettre en évidence les défauts géométriques d'un miroir ou les défauts de réfraction d'un verre par la faible déviation d'un rayon lumineux, Toepler, en 1864 , transpose l'idée à l'analyse des fluides présentant des variations d'indice. Mais c'est Ernst Mach qui, quelques années plus tard, inscrit l'optique dans l'histoire de la discipline grâce à ses études par imagerie des écoulements supersoniques. Les trois défauts rédhibitoires listés ci-dessus sont ainsi contournés de façon magistrale.

$\mathrm{Au} x \mathrm{xI} \mathrm{e}^{\mathrm{e}}$ siècle, ces principes restent à la base du succès de notre discipline, même si les moyens optiques employés n'ont plus grand-chose à voir avec les équipements de Mach.

\section{La place de la France sur l'échiquier mondial}

\section{Des nouvelles sont plutôt rassurantes}

Dans Photoniques $n^{\circ} 36$, nous avions exprimé nos regrets de voir la présence française se raréfier lors des grands événements internationaux de la spécialité, notamment le symposium ISFV, symposium majeur dans lequel nous étions les plus actifs (voir encadré 1). Le choix de ce critère est un autre débat, mais il a le mérite d'être simple à mettre en œuvre. Malgré les conséquences financières de la crise mondiale, qui ont incité les laboratoires à revoir leurs budgets de mission, la participation française s'est stabilisée en valeur absolue et améliorée en valeur relative. Lors du dernier symposium ISFV14 en Corée du Sud (Daegu, juin 2010), la France pointait en $5^{\mathrm{e}}$ position en nombre de communications (11 sur 243, à égalité avec la Russie), pour dix-neuf pays représentés. À noter que la première conférence invitée, traitant des méthodes exploitant la diffusion de type Rayleigh et Mie, était française.

\section{Nouvelle hiérarchie internationale quantitative}

L'encadré 1 montre la confirmation d'une nouvelle hiérarchie quantitative, déjà perceptible lors de ISFV 13 à Nice et évoquée dans notre éditorial de 2008.
Le centre de gravité s'est déplacé vers les pays asiatiques, même si la brutalité des chiffres est à tempérer en raison de la localisation du dernier symposium qui facilitait la présence des collègues coréens et japonais.

Avec 183 communications sur 243, le continent asiatique se taille la part du lion, malgré la faiblesse de la République Populaire de Chine. Toujours sur le plan quantitatif, le repli des États-Unis est un fait marquant, peut-être corrélé avec la baisse d'activité des grandes agences comme la Nasa dans le domaine des études aérospatiales. Toutefois, les critères qualitatifs placent toujours nos collègues américains au plus haut niveau, avec une position phare notamment dans le domaine de la nanofluidique. Globalement, I'Europe reste une valeur sûre avec 41 communications, la première place revenant à l'Allemagne avec 13 présentations.

\section{Et le club SFO «Fluvisu» - Diagnostic optique en mécanique des fluides?}

Créé en 1984, le comité national informel « Fluvisu », à l'origine « Visualisation en mécanique des fluides ", a été pérennisé en 2008/2009 par la SFO en tant que club « Diagnostic optique en mécanique des fluides ». Bien qu'indépendant du Club « CMOI - Contrôles et mesures opti-

Encadré 1.

ISFV 14 - International Symposium on Flow Visualization 14 - Daegu - Corée du Sud Juin 2010

Répartition géographique des intervenants :

\begin{tabular}{|l|c|}
\hline Japon & 84 \\
\hline Corée & 74 \\
\hline Allemagne et Taïwan & 13 \\
\hline Russie et France & 11 \\
\hline Grande-Bretagne & 9 \\
\hline Malaisie & 6 \\
\hline États-Unis & 4 \\
\hline Pays-Bas & 3 \\
\hline Biélorussie, Chine, Hongrie, & 2 \\
\hline Singapour, Suisse et Thaïlande & 1 \\
\hline Brésil, Grèce et Israël & 1
\end{tabular}

ISFV 13 avait été organisé à Nice en 2008 par le Comité Français "Fluvisu» et retournera en Asie en 2014 (Okinawa) après un passage en Biélorussie en 2012 (Minsk). ques pour l'industrie ", il est à noter que l'organisation des congrès nationaux a été fusionnée dès 2009, les deux communautés d'opticiens partageant à l'évidence les mêmes préoccupations.

La contribution française aux événements internationaux de type ISFV est essentiellement celle du club «Fluvisu». Par ailleurs, un bon indicateur de la santé de ce club est la proposition récente faite à son Président d'Honneur par la revue Optics and Laser in Engineering de préparer un numéro spécial consacré aux évolutions récentes de la discipline sous

\section{Encadré 2.}

Optics and Laser in Engineering - Special Issue 2011 - «Advances in Flow Visualization "

Manager Editor : Dario Ambrosini-Università I'Aquila- Italie

Guest Editor : Jean Pierre Prenel - Université de Franche Comté/Femto-ST

Dix articles de synthèse résument les avancées récentes du diagnostic optique dans des domaines variés d'applications faisant appel à la circulation de fluides. Un éditorial à caractère historique complète ces articles.

À paraître fin 2011 - Elsevier Publishing.

- Mesure des concentrations d'oxygène dans les écoulements liquides - Corée du Sud

- Évolution de l'imagerie strioscopique - États-Unis

- Applications pariétales des peintures sensitives - Japon

- Caractérisation des aérosols - Italie

- Écoulements solide/liquide en microfluidique - Corée du Sud

- Strioscopie couleur orientée vers l'arrière-plan - Allemagne et France

- Holographie numérique à trois couleurs - France

- Micro- et nanofluidique - Résonance de plasmons de surface - États-Unis

- Mesures hybrides : vélocimétrie et granulométrie simultanées de particules France

- Suivi de particules en microfluidique, écoulements sanguins - Corée du Sud

La faible participation du Japon, qui ne reflète pas l'activité réelle du pays, est directement liée au tremblement de terre catastrophique de mars 2011, qui a conduit à l'annulation de trois sujets qu'il convient de citer en raison de leur caractère d'actualité :

- Écoulements très rapides

- Écoulements polyphasiques

- Écoulements naturels 
le titre «Advances in Flow Visualization » (voir encadré 2). Rédigés à l'intention des opticiens non familiarisés avec la mécanique des fluides, dix articles de synthèse, dont trois rédigés par des auteurs du club «Fluvisu», constituent une bonne synthèse des progrès les plus récents.

\section{Les méthodes en 2011}

\section{Quel classement retenir?}

Pour les spécialistes mécaniciens, les modes de classement sont variés et complexes, péché mignon habituel de tous les spécialistes : type de fluide concerné (gazeux ou liquide, donc compressible ou non), qualitative ou quantitative, localisée ou plein champ - globale, 2D ou 3D, pariétale ou non, statique ou rapide... Pour l'opticien, le plus simple est de conserver la distinction traditionnelle entre les méthodes qui exploitent la diffusion de la lumière par des traceurs naturels ou introduits et celles qui traduisent les variations d'indice de réfraction en niveaux de lumière. La synthèse présen- tée en 2008 dans cette même revue restant d'actualité, le lecteur intéressé pourra s'y référer ( $n^{\circ} 36$, pages $52-57$ ).

\section{Quelle évolution depuis trois ans ?}

Comme indiqué en préambule, pas de rupture mais une progression constante vers plus de précision, de rapidité et de quantité d'informations. Les grandes tendances évoquées en 2008 ne font que se confirmer :

- L'introduction de la troisième dimension dans les visualisations et surtout dans les mesures s'est poursuivie, sur la base notamment de quelques idées majeures. Le couplage d'un éclairage tomographique (2D) et d'une observation stéréoscopique donne accès à la troisième composante de la vitesse (3C) au voisinage d'un plan. C'est l'approche 2D3C désormais classique.

Né vers 2005, le codage de la troisième composante de vitesse par la couleur est désormais opérationnel grâce aux progrès du traitement des images polychromatiques.
Par l'intermédiaire de sa variante numérique, I'holographie a fait un retour en force, notamment dans la caractérisation 3D des populations de particules.

La vélocimétrie de particules par corrélation de deux images successives (PIV pour les spécialistes - voir Photoniques $\left.n^{\circ} 36\right)$ couplée avec une photogrammétrie à triple imagerie conduit aussi à restituer un champ de vitesses à trois composantes.

Ces trois approches sont de type totalement 3D3C, et devraient évoluer vers des volumes de mesure plus larges et une plus grande quantité de traceurs analysés. Les progrès constants des sources laser et des procédés de traitement des images leur laissent encore une marge de manœuvre certaine.

L'exploitation des décalages Doppler induits par le mouvement des traceurs a poursuivi son développement et a donné lieu à des travaux de validation et de caractérisation en tant qu'outil métrologique. Rappelons que cette approche restitue un champ complet de $\gg$

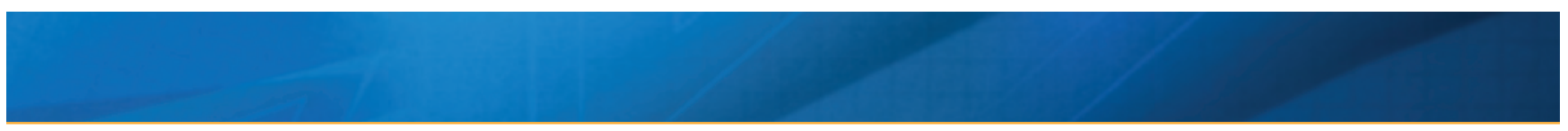

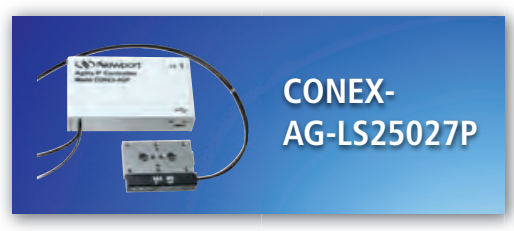
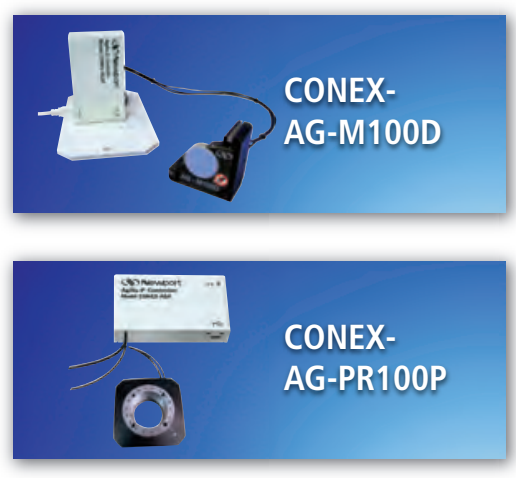

MICRO-CONTROLE Spectra-Physics S.A.S

1, rue Jules Guesde - Bâtiment B Tél.: 01.60.91.68.68

Zl. du Bois de l'Épine - BP189 Fax: 01.60.91.68.69

91006 Évry CEDEX

\section{CONEX}

\section{Nouveaux ensembles de micropositionnement intégrés.}

Une connexion aisée pour un système de contrôle simple.

Depuis l'arrivée de ses produits Agilis ${ }^{T M}$, Newport a poursuivi son avancée technologique de pointe en matière de moteurs piézoélectriques. Les innovations en termes de conception et de fabrication ont abouti à un ensemble de solutions à prix réduit, à haute résolution, en boucle fermée ou à positionnement absolu, utilisées dans le cadre de recherches avancées et idéales pour les applications OEM dans les domaines de la cytométrie, de la stabilisation de faisceau ultra rapide et de la recherche génomique. Grâce à nos contrôleurs CONEX ${ }^{\mathrm{TM}}$ intégrés et à notre plate-forme logicielle de gestion d'instruments améliorée NSTRUCT ${ }^{\mathrm{TM}}$, bénéficiez d'une configuration plus simple et d'un point de contrôle unique pour l'ensemble de vos besoins en matière d'instruments.

Si vous cherchez une solution de positionnement, faites confiance à Newport. Consultez www.newport.com/piezo-11 ou appelez-nous.

\section{MICRO-CONTROLE}

une marque de Newport Corporation

IIISTRUCT

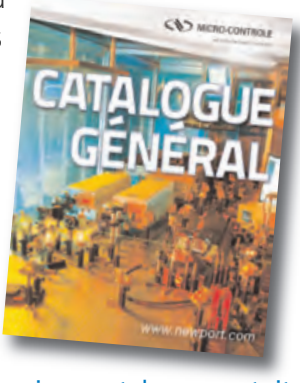

Pour recevoir un catalogue gratuit, inscrivez-vous sur

www.newport.com/resource2011
C 2011 Newport Corporation 
vitesses comme l'indique son étiquette de vélocimétrie Doppler globale.

- L'hybridation des méthodes de caractérisation des traceurs s'est fortement développée grâce à d'efficaces mariages de techniques : vélocimétrie et granulométrie ou vélocimétrie et estimation des températures par infrarouge, la tendance étant de passer de la mesure simultanée de deux paramètres à celle de trois. Ces hybridations pourraient sembler aisées à concrétiser par une simple association de montages optiques. II n'en est rien car les différents principes physiques sont souvent difficiles à concilier dans un même système : effet Doppler, fluorescence induite, rayonnement infrarouge, diffusion de type Rayleigh ou Mie. Ces dernières sont particulièrement performantes lorsque l'on sait exploiter astucieusement les paramètres de polarisation. On mesure ainsi le chemin parcouru depuis les premières visualisations tomographiques des années 1970 !

- L'utilisation des peintures sensitives, fournissant un signal surfacique chromatique lié à la température ou à la pression, a accédé au statut de méthode de mesure, la discrimination température/pression et la sensibilité ayant effectué des progrès conséquents depuis les premiers pas. Consacrée initialement aux caractérisations des interfaces de l'aéronautique, elle a donné lieu récemment à des applications originales comme la cartographie des disques durs tournant à grande vitesse en milieu confiné. Elle commence à migrer des parois vers le cœur des écoulements grâce à l'imprégnation des traceurs eux-mêmes. Une voie prometteuse vers une nouvelle hybridation vitesse/température.

- Enfin, on ne saurait oublier l'évolution des méthodes historiques comme l'interférométrie qui, avec le renfort des sources polychromatiques, fournissent

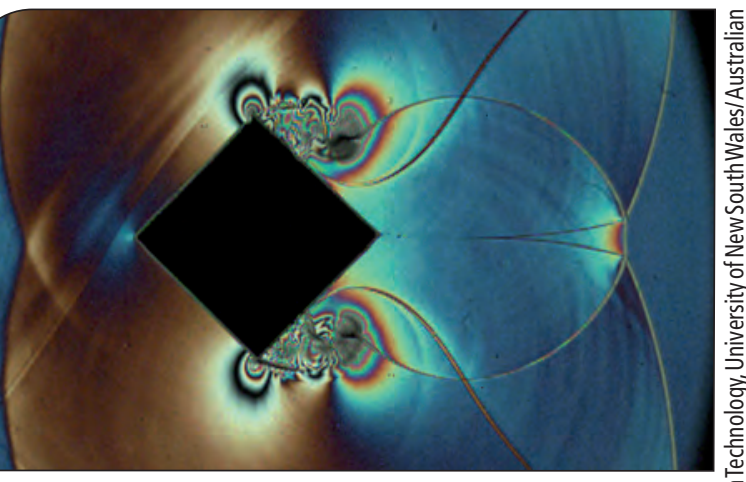

désormais de la gamme submicronique jusqu'à la pleine échelle des gros aéronefs.

Contrairement aux méthodes qui sont classées en fonction des lois de l'optique, pas de solution miracle pour classer simplement les innombrables applications. Ajoutons que le spectre de ces applications est devenu assez large pour considérer que tous les domaines d'activité humaine mettant en jeu un ou plusieurs fluides sont aujourd'hui concernés. L'approche la plus commune pour ébaucher un classement est donc celle des grands domaines de ces activités humaines : sciences physiques, technologie, sciences de la vie, sciences de la terre. À l'intérieur de ces grands domaines, on retrouvera les

Figure 1. Interférométrie polychrome : (a) interaction d'une onde de choc à Mach 1.32 avec un cylindre "diamant " ; (b) interaction d'une onde de choc à Mach 1.32 avec un cylindre.

de plus en plus d'information sur les écoulements compressibles, tout en étant capables de surveiller les mouvements ou déformations des interfaces (figure 1). Une autre forme d'hybridation entre approches fluide et pariétale.

\section{Les domaines d'application 2011}

\section{Quel classement retenir?}

Comme pour les méthodes, les modes de classement des spécialistes sont nombreux et dépendent du domaine d'activité : fluide compressible ou incompressible, mono- ou polyphasique, isothermique ou non, statique ou instationnaire, échelles des dimensions et gammes des vitesses... Dans ce dernier domaine, la distinction simplifiée «sub, trans, supersonique" qui était liée à la prépondérance des applications aérodynamiques pour véhicules terrestres ou aériens est aujourd'hui dépassée puisque la gamme s'étend désormais de quelques $\mu \mathrm{m} / \mathrm{s}$ en microfluidique à Mach 6/8 en vol hypersonique. Même constatation pour les échelles, qui vont listes des spécialités plus ou moins normalisées, par exemple en technologie: transports (eux-mêmes scindés en terrestres, maritimes ou aériens), énergie (thermique, électrique), etc. Une synthèse exhaustive sort donc totalement du cadre de cet article.

\section{Quelques points forts et nouvelles pistes}

- Le domaine historique de l'aérodynamique des transports reste plus que jamais d'actualité. L'aérodynamique exceptionnelle de l'Airbus 380 (comment rendre stable une telle masse sans utiliser des dimensions rédhibitoires ?) a nécessité de longues et complexes expérimentations en soufflerie. Après une phase de stagnation relative post-choc pétrolier, l'aérodynamique automobile est en ébullition afin de chasser les grammes de $\mathrm{CO}_{2}$ superflus et de passer sous les paliers fatidiques de la réglementation européenne. La moindre traînée marginale est pourchassée : rétroviseurs, entrées d'air, passages de roue, essuie-glaces... Les mesures optiques sont omniprésentes dans toutes ces activités.

- Le domaine également ancien de la combustion n'est pas en reste dans ce contexte. Un exemple spectaculaire est 
celui des chambres de combustion des moteurs thermiques : révolution en diesel avec la génération «rampe commune haute pression ", révolution dans les moteurs à allumage commandé avec la généralisation de l'injection directe et bientôt apparition du taux de compression variable ou des moteurs mixtes alliant le rendement du diesel à la propreté chimique de l'essence...

La mise au point des indispensables modèles de combustion doit beaucoup à l'optique qui traque les microgouttelettes de carburant en 3D dans des conditions extrêmes de température et de pression (voir le dossier « Diagnostics optiques pour les moteurs automobiles " dans Photoniques $n^{\circ} 52$ ).

- Les sciences de la terre avaient déjà été citées en 2008 comme un domaine prometteur. Grandes consommatrices de modèles, elles font appel massivement aux capteurs localisés multiples et aux télémesures satellitaires dans les études à échelle réelle. En revanche, les expérimentations à échelle réduite, très répandues notamment dans le domaine de I'hydraulique (bassins, tunnels et plaque tournante Coriolis) associent le plus souvent les capteurs traditionnels et les mesures optiques.

La simulation des vagues et des courants n'est pas nouvelle mais a connu un regain d'intérêt ces dernières années en raison des préoccupations environnementales. Une application spectaculaire a récemment vu le jour, notamment en France : la simulation des fameuses "vagues scélérates» variantes liquides de nos solitons optiques. Ce point se devait de figurer dans une revue d'opticiens car le parallèle soliton optique/vague solitaire a permis d'associer les deux communautés au-delà de la simple utilisation des mesures optiques.

- Les sciences de la vie ont fait une entrée remarquée à travers les mesures opti ques en microfluidique et les applications biomédicales, aujourd'hui routinières dans les laboratoires d'analyse. Le spectre de ces applications s'élargit désormais régulièrement : écoulements sanguins, observation et manipulation de cellules vivantes dans des fluides... La microsco-

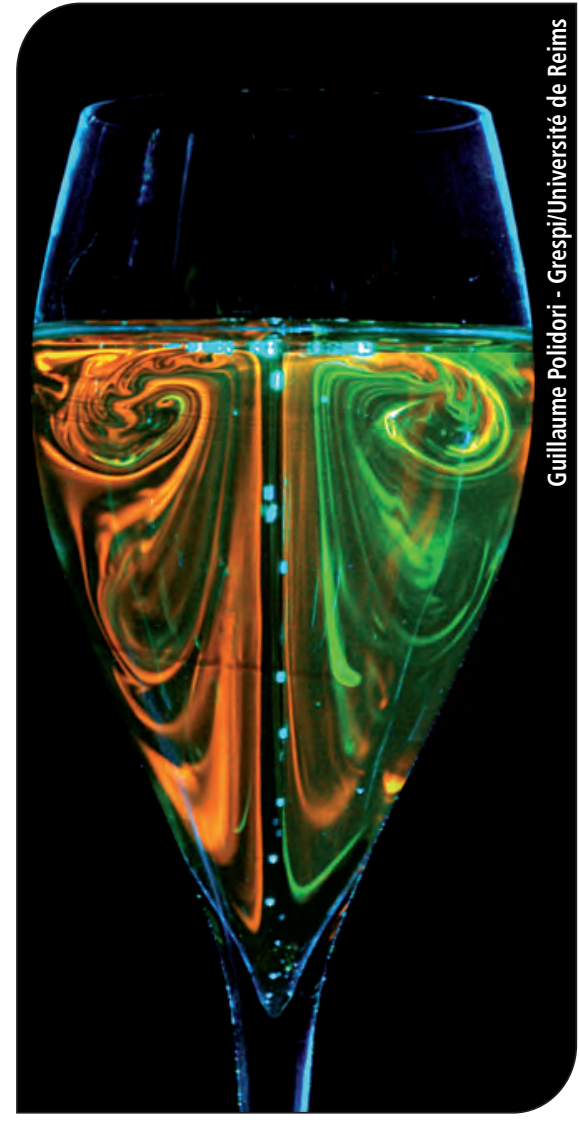

Figure 2. Tomographie avec traceurs fluorescents polychromes : étude de la corrélation entre saveur/fragrance et mouvements convectifs. Mise en évidence des rôles de la forme et de l'état de surface du verre.

pie et ses perfectionnements sophistiqués s'associent désormais avec des méthodes de caractérisation des écoulements comme la vélocimétrie. La frontière entre les deux domaines devient d'ailleurs impossible à définir.

Plus «terre à terre », mais importante par ses retombées commerciales et festives, I'optimisation des vins de Champagne grâce aux méthodes tomographiques a fait son apparition récemment (figure 2). Les bulles sont utilisées depuis longtemps comme traceurs artificiels dans les écoulements liquides (générateurs électrolytiques ou chimiques). Il suffisait de penser à utiliser les traceurs naturels présents dans nos champagnes! Personne dans la communauté internationale ne s'est étonné que l'idée soit venue des Français... du côté de Reims. Le fait que l'actuel président du club «Fluvisu » soit un universitaire rémois n'est que le fruit d'une coïncidence...
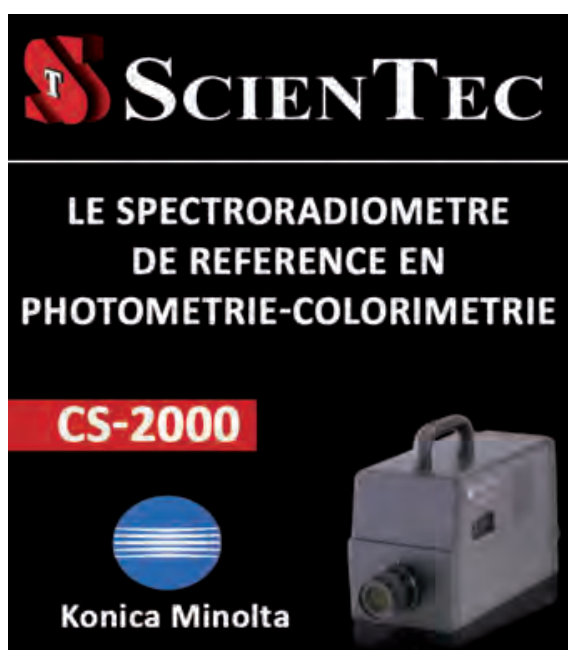

CARACTERISTIQUES

- 3 ANGLES DE MESURE $\left(1^{\circ}, 0.2^{\circ}, 0.1^{\circ}\right)$

- COMPACT

- MESURE RAPIDE

- HAUTE SENSIBILITE

(mesure à partir de

$0.003 \mathrm{~cd} / \mathrm{m}^{2}$ avec version

CS-2000 et $0.0005 \mathrm{~cd} / \mathrm{m}^{2}$ avec version CS-2000A)

- MESURE DE CONTRASTE jusqu'à 1:100 000

- MODE AUTONOME

(avec écran de visualisation) ou utilisation avec ordinateur (par liaison USB)

FOURNIT

la luminance,

la couleur,

le spectre (380-780 nm),

la température de couleur,

la longueur d'onde dominante,

I'indice de rendu des couleurs (IRC)

\section{ScienTec c'est aussi ...}

Sources de référence

Vidéocolorimètres

Photomètres

Luxmètres

Chromamètres

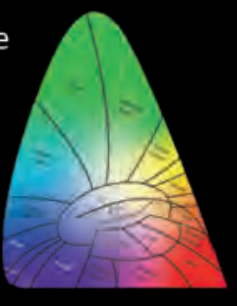

Tel : +33 (0) 1.64.53.27.00

info@scientec.fr / www.scientec.fr 